\title{
Effects of clinicopathological factors on prognosis of young patients with breast cancer
}

\author{
Wen Li ${ }^{1,2, *}$, Guangzhi Ma ${ }^{2, *}$, Qiang Wu ${ }^{2, *}$, Ya Liu ${ }^{1}$, Xuejuan Liu ${ }^{1}$ and Jing Wang ${ }^{1}$ \\ ${ }^{1}$ Department of Thyroid and Breast Surgery, West China Hospital of Sichuan University, Chengdu, Sichuan 610041, P. R. \\ China \\ ${ }^{2}$ Lung Cancer Center, West China Hospital of Sichuan University, Chengdu, Sichuan 610041, P. R. China \\ *These authors contributed equally to this work
}

Correspondence to: Jing Wang, email: wangj889@163.com

Keywords: young age; breast cancer; prognosis; molecular subtypes; survival

Received: July 05, $2017 \quad$ Accepted: December 30, $2017 \quad$ Published: January 06, 2018

Copyright: Li et al. This is an open-access article distributed under the terms of the Creative Commons Attribution License 3.0 (CC BY 3.0), which permits unrestricted use, distribution, and reproduction in any medium, provided the original author and source are credited.

\section{ABSTRACT}

Objective: This study aims to analyze the relationship between clinicopathological characteristics and survival in young patients ( $\leq 35$ years old) with breast cancer.

Results: Compared with patients aged $\mathbf{3 0}$ to $\mathbf{3 5}$ years, age of younger patients ( $\leq 30$ years) was an independent predictor for poor disease-free survival (DFS) and overall survival (OS). PR negative status $(p=0.042)$, high tumor grade $(p=0.012)$, and advanced lymph nodes post-surgery $(p<0.001)$ were independent prognostic factors of DFS, while PR negative status $(p=0.003)$ and advanced lymph nodes post-surgery $(p=0.002)$ were both independent prognostic factors of OS. For patients with hormone receptor-positive breast cancer, people with ER+ or PR+ and HER2-/+ status showed poorer prognosis than the other two levels. Risk factor grouping based on the ER, PR, HER2, Ki-67 status, tumor grade, and lymph nodes post-surgery showed that patients in highest score group received the poorest prognosis.

Materials and Methods: A total of 173 cases of young breast cancer patients were included in this study. The clinicopathological factors potentially associated with prognosis were evaluated by univariate and multivariate analyses. Furthermore, we categorized patients into different groups to evaluate the prognosis according to hormone receptor status or important risk factors.

Conclusions: Patient age, PR status, tumor grade, and lymph nodes post-surgery had clinical value as predictive factors of prognosis. Grading system based on the hormone status or the risk factor grouping may offer a useful approach to assess which subgroups of young breast cancer patients present poorer prognosis.

\section{INTRODUCTION}

Breast cancer, one of the most commonly diagnosed cancer in women worldwide, affects more than 1.3 million individuals and accounts for about $14 \%$ of cancer-related deaths $[1,2]$. Approximately $6.6 \%$ of breast cancer patients are diagnosed in women younger than 40 years, $2.4 \%$ in those younger than 35 , and $0.65 \%$ in those younger than 30 [3]. However, in China, the reported cases in women younger than 35 years are markedly higher than those in western countries $[4,5]$. Moreover, the increase in young breast cancer patients is highly problematic, and the behavior of these tumors is more aggressive [6]. Young women with breast cancer are likely to present advanced stages at diagnosis, including higher histologic grade, larger tumor size, more aggressive pathological characteristics, and higher rates of recurrence at any clinical stage in comparison with their older counterparts [7]. Although several large-scale studies have reported that young age $(\leq 35)$ is an independent prognostic factor for both disease-free survival (DFS) and overall survival (OS) $[8,9]$, few studies focused on the impact of clinical and pathologic factors on the prognosis in young breast cancer patients. This study aimed to evaluate the prognostic 
significance of clinicopathological factors stratified by age, surgery type, molecular subtype based on estrogen receptor (ER), progesterone receptor (PR), and human epidermal growth factor receptor 2 (HER2) statuses. In addition, considering that hormone receptor-positive breast cancer is the main subtype in young patients, we divided hormone receptor-positive breast cancer into different groups to assess the prognostic influence on young patients.

\section{RESULTS}

\section{Clinicopathological factors and outcome}

According to the 2013 St. Gallen expert consensus, the molecular subtypes of all the 173 cases were divided into 5 types: 27 cases were classified into luminal A subtype (15.6\%), 57 cases into Luminal B (HER2-) subtype (32.9\%), 38 cases into Luminal B (HER2+) subtype (22.0\%), 19 cases into HER2 subtype (11.0\%), and 32 cases in to TNBC subtype (18.5\%), with $128(74.0 \%)$ patients undergoing mastectomy while 45 (26.0\%) patients received breast-conserving surgery (BCS). The median age of all patients was 32, within median follow-up time of 64 months. Tumor relapse occurred in 59 cases, among which 42 cases died. The clinical and pathologic characteristics of the 173 patients included in this study are shown in Table 1. In univariate analysis, PR status, tumor grade, lymph nodes post-surgery, and histological grade were significantly associated with DFS and OS. In addition, patients younger than 30 was associated with significantly shorter DFS and OS compared with patients aged from 30 to 35 ( $p=0.019$ and $p=0.011$, respectively, Table 1 , Figure 1A, 1B). As shown in Table 4, in multivariate analysis, patient age ( $p=$ $0.002)$, PR status $(p=0.042)$, tumor grade $(p=0.012)$, and lymph nodes post-surgery $(p<0.001)$ were independent predictors of DFS for young patients, whereas patient age $(p=0.002)$, PR status $(p=0.003)$, and lymph nodes post-surgery $(p=0.002)$ were correlated with OS. In both univariate and multivariate analyses, the classification of patient age was an independent predictor for DFS and OS (Figure 1A and 1B and Tables 2 and 4), indicating that patients younger than 30 shows a poorer prognosis compared with patients aged 31 to 35 . We analyzed the clinical and pathologic factors between the two groups at diagnosis, as shown in Table 2, the expression levels of ER, PR, HER2, and Ki-67, tumor size, lymph nodes postsurgery, molecular subtype, and treatment condition did not display any difference between women aged 31 to 35 and women younger than 30 years of age.

\section{Local therapy, age, and survival}

We evaluated the effect of surgery type, including BCS and mastectomy, on DFS and OS in young patients with T1-T2N0-N+M0 breast cancer. A total of 146 patients were considered, with 101 patients undergoing mastectomy and 45 undergoing BCS. No connection was observed between surgery type and cumulative probability of DFS $(P=0.120)$ or OS $(P=0.140)$. Moreover, the effectiveness of surgery type stratified by age on DFS and OS were analyzed. Compared with patients aged 30-35 years, patients below 30 years of age were associated with lower DFS after mastectomy $(P=0.007)$ but not associated with OS $(P=0.204)$. No statistically significant difference in both DFS and OS after BCS was observed between patients aged form 31 to 35 and patients younger than 30 years old $(P=0.345$, and $P=0.755$, respectively).

\section{Prognosis and receptor-positive breast cancer or risk factors grouping}

The hormone receptor levels were 1, 2, and 3 in 59 (48.4\%), 24 (19.7\%), and 39 (32.0\%) of the 122 patients with hormone receptor-positive breast cancer, respectively. In univariate analysis, hormone receptor level was an independent prognostic factors for both DFS and OS $(p=0.002$; Figure 2A, 2B, Table 3). Young breast cancer patients with ER+, PR+, and HER2- received the best prognosis, whereas the group of ER+ or PR + , HER2-/+ patients had the poorest prognosis in hormone receptorpositive patients. In the present study, the risk factor group based on ER, PR, HER2, and Ki-67 status, tumor grade, and lymph nodes post-surgery were 1, 2, and 3 in 125 $(72.3 \%), 38(22 \%)$, and $10(5.8 \%)$ of the 173 patients, respectively. Patients in risk group 3 were predicted to present the poorest DFS and OS $(p<0.001$; Figure 3A, $3 \mathrm{~B}$, Table 3 ). All of the 10 patients in group 3 progressed, and 8 patients died. In multivariate analysis, hormone receptor level was an independent prognostic factor for DFS $(p=0.038)$, and the risk factor group was an interrelated prognostic predictor of DFS and OS in young patients ( $p=0.006$, and $p=0.010$, respectively; Table 4$)$.

\section{DISCUSSION}

Previous studies have shown that young age $(\leq 35$ years old) is an adverse prognostic factor for women with breast cancer; moreover, compared with older patients, younger breast cancer patients present later disease stage, higher grade tumors (poorer and more undifferentiated tumors), and poorer receptor status [13, 14]. However, relatively few studies focused on the prognostic effect of clinicopathologic factors among young breast cancer patients. In the present study, we selected several common clinical and pathological factors, including patient age, ER, PR, HER2, Ki-67 status, tumor histological grade, tumor grade, lymph nodes post-surgery, molecular subtype, and treatment condition, which were possible predictors of cancer outcomes. The results implied that patient age, PR status, tumor grade, and lymph nodes post-surgery were associated with DFS, whereas patient age, PR status, 



Figure 1: Kaplan-Meier survival analyses of correlations between age and survival among breast cancer patients. PFS (A) and OS (B). 

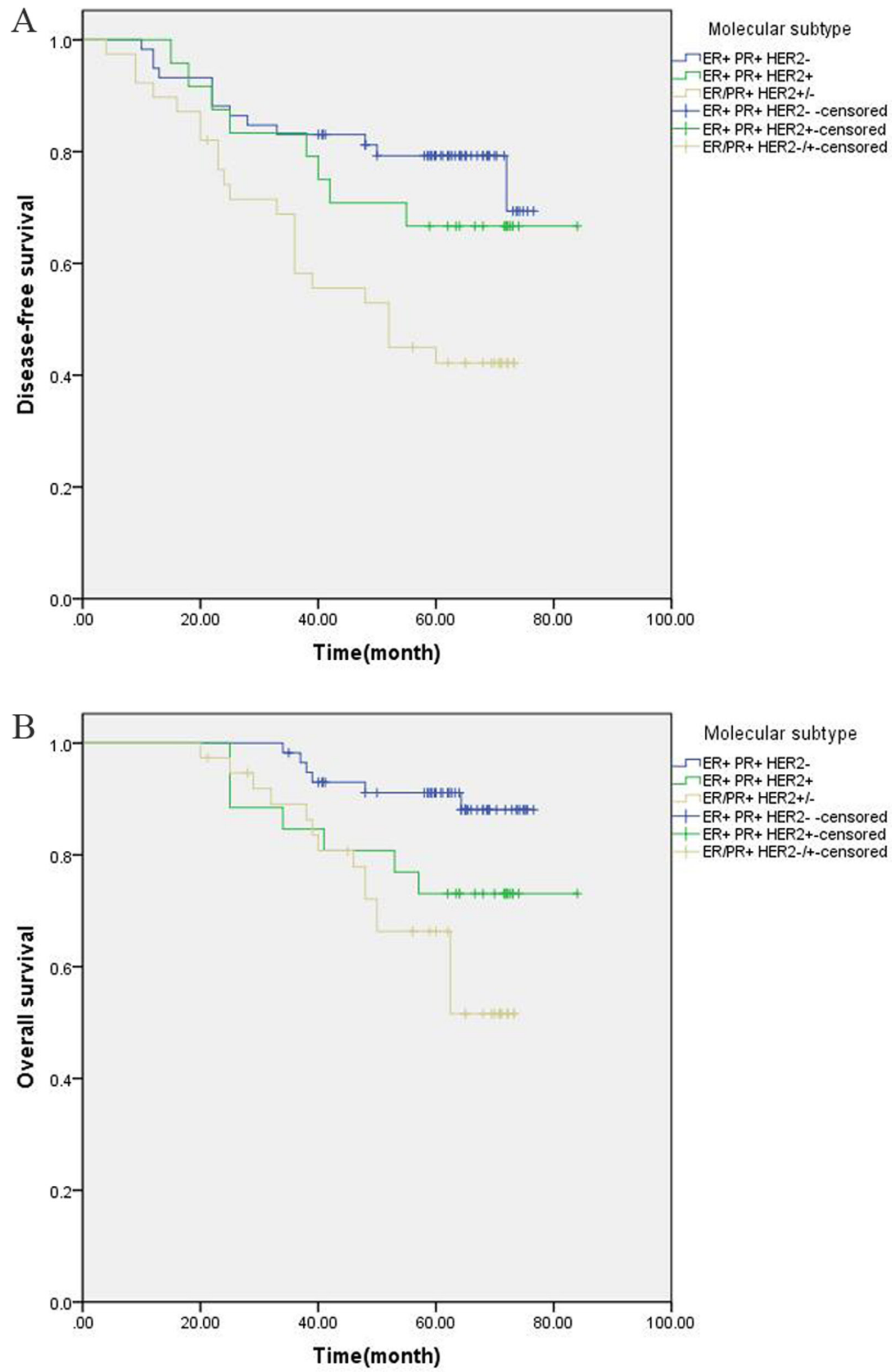

Figure 2: ER, PR, HER2 status and survival. Kaplan-Meier survival analysis on DFS (A) in accordance with ER, PR, and HER2 status. Kaplan-Meier survival analysis on OS (B) in accordance with ER, PR, and HER2 status. 

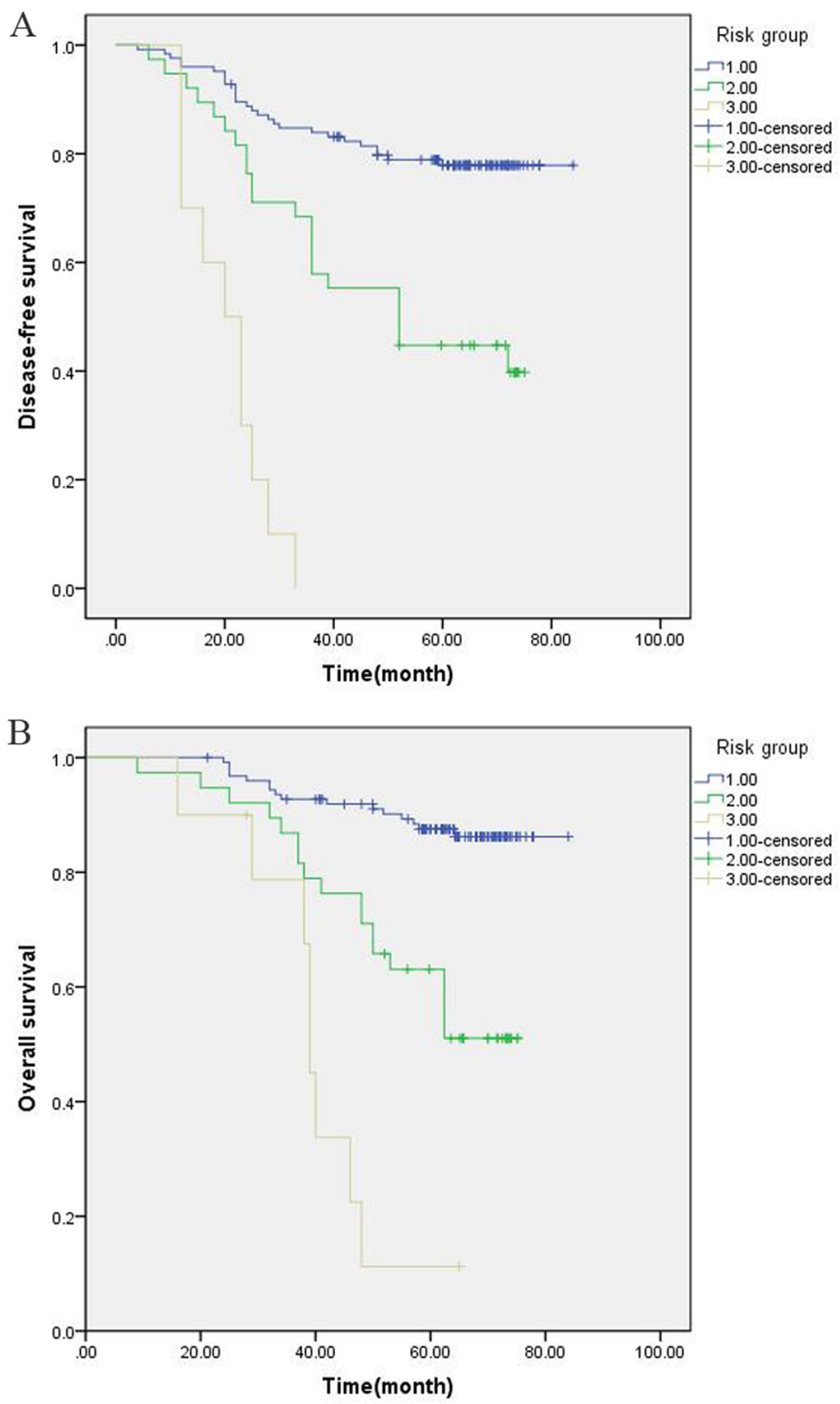

Figure 3: Risk factor grouping and survival. DFS by Kaplan-Meier survival analysis (A) in accordance with risk factor grouping. OS by Kaplan-Meier survival analysis (B) in accordance with risk factor grouping. 
and lymph nodes post-surgery were associated with OS. Older age (30-35) suggested better prognosis, whereas PR negative status, large tumor grade, and advanced lymph nodes post-surgery were associated with poorer prognosis.

It has been generally recognized that young age itself is an independent risk factor for recurrence and death. A study based on a national population cancer registry showed that young age is an independent risk factor for death $(\mathrm{HR}=1.095)$ [15]. Peng et al. [16] retrospectively analyzed a large cohort of 511 young breast cancer patients aged $\leq 35$ years by comparing clinicopathological characteristics with a cohort of 551 older patients aged from 35 to 50 years old, and their results showed that younger patients present significantly shorter DFS than their older counterparts (median 23.2 months versus 28.4 months, $p=0.024$ ). However, few studies discussed the prognostic effect of age in young breast cancer patients. A trial referring to age $\leq 40$ years as young breast cancer revealed that patient age of younger than 35 years (as opposed to 35-40) was an independent risk factor for poorer local relapse-free survival, DFS, and OS [17]. Moreover, Zhao et al. [3] reported that patients who were younger than 30 presented poorer prognosis compared with patients aged from 31 to 35 . In our study, patient age showed a significant correlation with both DFS and OS in young patients, and patients younger than 30 years of age showed an adverse prognosis compared with patients aged from 31 to 35; this finding was consistent with those of the previous study. Moreover, the results showed no difference between age and clinicopathological factors in the two groups when classified by age; again, these results were consisted with those of a previous study [17].

Given that young women with breast cancer are known to have a higher risk of local recurrence and mortality, the impact of surgical treatment on outcomes in young breast cancer patients remains unclear, and $\mathrm{BCS}$ in young patients remains to be a controversial issue [18]. Several studies suggested that young women (younger than 35 or 40 years) show inferior cosmetic outcomes with BCS, implying that these women may be better served by mastectomy [19]. However, Quan et al. [18] reported similar oncologic outcomes in a cohort of young women $(\leq 35)$ who were selected for treatment with BCS and mastectomy. Besides, an meta-analysis carried out on 22598 patients showed that mastectomy unlikely provides better OS compared to BCS in early young breast cancer patients [20]. In the present study, our results demonstrated no differences in DFS and OS between BCS and mastectomy in young breast cancer patients. When surgery type was stratified by age, compared with patients aged from 30 to 35 years, patients aged $\leq 30$ years was associated with lower DFS after mastectomy but not associated with OS. These results may suggest that BCS is a rational option as surgery type for young women. However, patients included in this cohort had 84.4\% T1/
T2 tumors, but only 45 cases $(26.0 \%)$ received BCS. A nationwide survey indicated that in developed urban areas in China, BCS were performed on only $24.3 \%$ in 2008 , which is much lower compared with western countries [21]. One explanation for this is lack of resources for radiation therapy, especially in less developed regions in China [22]. Hence readers should be cautious referring to the result considering the limited patient number that received $\mathrm{BCS}$.

A previous study observed that a negative PR status occurs more frequently in young breast cancer patients [23]. In addition, PR is known to be regulated by the estrogen receptor $\alpha(\mathrm{ER} \alpha)$, and loss of PR expression indicates a more aggressive disease phenotype, which is less dependent on estrogen signaling [11]. Mohammed et al. reported that PR lacks proliferative potential; importantly $\mathrm{PR}$ is an anti-proliferative factor in an estrogen-driven context [24]. Besides, PR+ status distinctly improves outcome prediction over ER status alone for patients who receive adjuvant endocrine therapy, and several studies confirmed that the absence of PR is an independent risk factor for DFS or OS [25]. In our study, we detected that PR negative status was an independent risk factor and was significantly associated with a more unfavorable DFS and OS in both univariate and multivariate analyses. By contrast, ER expression was not typically related with prognosis, as was consistent with the results of previous studies.

The molecular subtypes of breast cancer are considered to reflect tumor biology and exert a vital influence on prognosis. Patients with luminal A subtype are likely to receive the best prognosis than the other three subtypes [26]. The proportion distribution of molecular subtypes is different in young breast cancer patients and, young breast cancer patients present a higher rate of triple-negative and HER2 overexpression subtypes compared with the older patients [15]. Villarreal-Garza $\mathrm{C}$ et al. [27] reported that young women diagnosed with hormone receptor-positive breast cancer in Mexico were more specifically categorized as luminal B, experiencing poor survival outcomes. In our study, IHC surrogate for luminal B was the most common, account for more than half of all cases. Moreover, considering that hormone receptor-positive breast cancer remains to be the major subtype among young patients [28], we categorized the hormone-positive breast cancer into three levels, and the results showed that $\mathrm{ER}+, \mathrm{PR}+, \mathrm{HER} 2-$ showed better prognosis compared with the other two kinds, and ER+ or $\mathrm{PR}+, \mathrm{HER} 2-/+$ exhibited the poorest prognosis among the three subtypes.

ER, PR, HER2, and Ki-67 status, tumor grade, and axillary lymph nodes involved post-surgery are combined to determine a prognostic parameter [3]. In our study, the risk factor-based combination classification were divided into three groups, and the results revealed that the risk score group was an independent prognostic predictor, with 
Table 1: Clinicopathological characteristics and outcomes among young breast cancer patients

\begin{tabular}{|c|c|c|c|c|c|c|c|}
\hline & Total & Relapse & Death & & DFS & & OS \\
\hline & & & & $p$ & HR $(95 \% \mathrm{CI})$ & $p$ & HR $(95 \% \mathrm{CI})$ \\
\hline Patient age & & & & 0.019 & $0.539(0.322-0.901)$ & 0.011 & $0.467(0.255-0.857)$ \\
\hline$\leq 30$ & $57(32.9 \%)$ & $26(44.1 \%)$ & $20(47.6 \%)$ & & & & \\
\hline $30-35$ & $116(67.1 \%)$ & $33(55.9 \%)$ & $22(52.4 \%)$ & & & & \\
\hline Family history & & & & 0.586 & $1.874(0.928-3.785)$ & 0.762 & $2.056(0.894-4.731)$ \\
\hline Yes & $27(15.6 \%)$ & $10(16.9 \%)$ & $7(16.7 \%)$ & & & & \\
\hline No & $146(84.4 \%)$ & $49(83.1 \%)$ & $35(83.3 \%)$ & & & & \\
\hline ER & & & & 0.296 & $1.358(0.765-2.410)$ & 0.890 & $1.047(0.544-2.105)$ \\
\hline+ & $117(67.6 \%)$ & $43(72.9 \%)$ & $29(69.0 \%)$ & & & & \\
\hline - & $56(32.4 \%)$ & $16(27.1 \%)$ & $13(31.0 \%)$ & & & & \\
\hline PR & & & & 0.031 & $1.789(1.055-3.030)$ & 0.003 & $2.786(1.427-5.435)$ \\
\hline+ & $88(50.9 \%)$ & $22(37.3 \%)$ & $12(28.6 \%)$ & & & & \\
\hline - & $85(49.1 \%)$ & $37(62.7 \%)$ & $30(71.4 \%)$ & & & & \\
\hline HER2 & & & & 0.813 & $0.936(0.544-1.612)$ & 0.532 & $1.207(0.669-2.178)$ \\
\hline+ & $43(24.9 \%)$ & $13(22.0 \%)$ & $11(26.2 \%)$ & & & & \\
\hline- & $130(75.1 \%)$ & $46(78.0 \%)$ & $31(73.8 \%)$ & & & & \\
\hline Ki-67 status & & & & 0.451 & $1.253(0.697-2.251)$ & 0.396 & $1.360(0.668-2.766)$ \\
\hline+ & $120(69.4 \%)$ & $44(74.6 \%)$ & $32(76.2 \%)$ & & & & \\
\hline- & $53(30.6 \%)$ & $15(25.4 \%)$ & $10(23.8 \%)$ & & & & \\
\hline pT Stage & & & & $<0.001$ & $2.020(1.507-2.708)$ & $<0.001$ & $2.148(1.525-3.026)$ \\
\hline 1 & $65(37.6 \%)$ & $9(15.3 \%)$ & $5(11.9 \%)$ & & & & \\
\hline 2 & $81(46.8 \%)$ & $34(57.6 \%)$ & $25(59.5 \%)$ & & & & \\
\hline 3 & $22(12.7 \%)$ & $13(22.0 \%)$ & $9(21.4 \%)$ & & & & \\
\hline 4 & $5(2.9 \%)$ & $3(5.1 \%)$ & $3(7.1 \%)$ & & & & \\
\hline pN Stage & & & & $<0.001$ & $2.410(1.837-3.160)$ & $<0.001$ & $2.483(1.814-3.399)$ \\
\hline 0 & $75(43.4 \%)$ & $13(22.0 \%)$ & $10(23.8 \%)$ & & & & \\
\hline 1 & $52(30.1 \%)$ & $15(25.4 \%)$ & $6(14.3 \%)$ & & & & \\
\hline 2 & $33(19.1 \%)$ & $19(32.2 \%)$ & $16(38.1 \%)$ & & & & \\
\hline 3 & $13(7.5 \%)$ & $12(20.3 \%)$ & $10(23.8 \%)$ & & & & \\
\hline Molecular subtype & & & & 0.672 & $1.165(0.685-1.826)$ & 0.353 & $1.321(0.565-2.842)$ \\
\hline Luminal A & $27(15.6 \%)$ & $8(13.6 \%)$ & $5(11.9 \%)$ & & & & \\
\hline Luminal B (HER2-) & $57(32.9 \%)$ & $19(32.2 \%)$ & $13(31.0 \%)$ & & & & \\
\hline Luminal B (HER2+) & $38(22.0 \%)$ & $14(23.7 \%)$ & $10(23.8 \%)$ & & & & \\
\hline $\begin{array}{l}\text { HER2- } \\
\text { enriched }\end{array}$ & $19(11.0 \%)$ & $7(11.8 \%)$ & $5(11.9 \%)$ & & & & \\
\hline TNBC & $32(18.5 \%)$ & $11(18.6 \%)$ & $9(21.4 \%)$ & & & & \\
\hline Histological grade & & & & $<0.001$ & $2.801(2.134-3.284)$ & $<0.001$ & $2.369(1.365-3.956)$ \\
\hline I-II & $118(68.2 \%)$ & $33(55.9 \%)$ & $20(47.6 \%)$ & & & & \\
\hline III & $55(31.8 \%)$ & $26(44.1 \%)$ & $22(52.4 \%)$ & & & & \\
\hline Surgery type & & & & & $0.6201 .134(0.634-2.028)$ & 0.509 & $1.259(0.636-2.494)$ \\
\hline Mastectomy & $128(74.0 \%)$ & $44(74.6 \%)$ & $32(76.2 \%)$ & & & & \\
\hline BCS & $45(26.0 \%)$ & $15(25.4 \%)$ & $10(23.8 \%)$ & & & & \\
\hline Chemotherapy & & & & & $0.4571 .304(0.649,2.647)$ & 0.732 & $1.149(0.519,2.647)$ \\
\hline Yes & $143(82.7 \%)$ & $47(79.7 \%)$ & $35(83.3 \%)$ & & & & \\
\hline No & $30(17.3 \%)$ & $12(20.3 \%)$ & $7(16.7 \%)$ & & & & \\
\hline Hormone therapy & & & & & $0.3471 .365(0.713,2.613)$ & 0.435 & $1.345(0.695-2.935)$ \\
\hline Yes & $137(79.2 \%)$ & $49(83.1 \%)$ & $35(83.3 \%)$ & & & & \\
\hline No & $36(20.8 \%)$ & $10(16.9 \%)$ & $7(16.7 \%)$ & & & & \\
\hline Radiotherapy & & & & & $0.8811 .041(0.634,1.733)$ & 0.451 & $1.262(0.682,2.215)$ \\
\hline Yes & $82(47.4 \%)$ & $28(47.4 \%)$ & $19(86.3 \%)$ & & & & \\
\hline No & $91(52.6 \%)$ & $31(52.5 \%)$ & $23(54.8 \%)$ & & & & \\
\hline Target therapy & & & & & $0.7921 .103(0.534,2.278)$ & 0.547 & $1.303(0.559,3.079)$ \\
\hline Yes & $25(14.4 \%)$ & $9(15.2 \%)$ & $7(16.7 \%)$ & & & & \\
\hline No & $148(85.6 \%)$ & $50(84.7 \%)$ & $35(83.3 \%)$ & & & & \\
\hline
\end{tabular}


Table 2: Patient and tumor characteristics by age group

\begin{tabular}{|c|c|c|c|c|}
\hline & Total & $\leq \mathbf{3 0}$ & $30-35$ & $P$ \\
\hline Family history & & & & 0.623 \\
\hline Yes & $27(15.6 \%)$ & $10(17.5 \%)$ & $17(14.7 \%)$ & \\
\hline No & $146(84.4 \%)$ & $47(82.5 \%)$ & $99(85.3 \%)$ & \\
\hline ER & 173 & 57 & 116 & 0.397 \\
\hline+ & $117(67.6 \%)$ & $41(71.9 \%)$ & $76(65.5 \%)$ & \\
\hline- & $56(32.4 \%)$ & $16(28.1 \%)$ & $40(34.5 \%)$ & \\
\hline PR & & & & 0.999 \\
\hline+ & $88(50.9 \%)$ & $29(50.9 \%)$ & $59(50.9 \%)$ & \\
\hline- & $85(49.1 \%)$ & $28(49.1 \%)$ & $57(49.1 \%)$ & \\
\hline HER2 & & & & 0.116 \\
\hline+ & $42(24.3 \%)$ & $18(31.6 \%)$ & $24(20.7 \%)$ & \\
\hline- & $131(75.7 \%)$ & $39(68.4 \%)$ & $92(79.3 \%)$ & \\
\hline Ki-67 status & & & & 0.055 \\
\hline+ & $120(69.4 \%)$ & $45(78.9 \%)$ & $75(64.7 \%)$ & \\
\hline- & $53(30.6 \%)$ & $12(21.1 \%)$ & $41(35.3 \%)$ & \\
\hline pT Stage & & & & 0.778 \\
\hline 1 & $65(37.6 \%)$ & $29(50.9 \%)$ & $60(51.7 \%)$ & \\
\hline 2 & $81(46.8 \%)$ & $21(36.8 \%)$ & $36(31.0 \%)$ & \\
\hline 3 & $22(12.7 \%)$ & $6(10.5 \%)$ & $16(13.8 \%)$ & \\
\hline 4 & $5(2.9 \%)$ & $1(1.8 \%)$ & $4(3.4 \%)$ & \\
\hline pN Stage & & & & 0.635 \\
\hline 0 & $75(43.4 \%)$ & $28(49.1 \%)$ & $47(40.5 \%)$ & \\
\hline 1 & $52(30.1 \%)$ & $15(26.3 \%)$ & $37(31.9 \%)$ & \\
\hline 2 & $33(19.1 \%)$ & $9(15.8 \%)$ & $24(20.7 \%)$ & \\
\hline 3 & $13(75.1 \%)$ & $5(8.8 \%)$ & $8(6.9 \%)$ & \\
\hline Molecular subtype & & & & 0.887 \\
\hline Luminal A & $27(15.6 \%)$ & $8(14.0 \%)$ & $20(17.2 \%)$ & \\
\hline Luminal B (HER2-) & $57(32.9 \%)$ & $17(29.8 \%)$ & $36(31.0 \%)$ & \\
\hline Luminal B (HER2+) & $38(22.0 \%)$ & $15(26.3 \%)$ & $25(21.6 \%)$ & \\
\hline $\begin{array}{l}\text { HER2- } \\
\text { enriched }\end{array}$ & $19(57.8 \%)$ & $6(10.5 \%)$ & $9(7.8 \%)$ & \\
\hline TNBC & $32(18.5 \%)$ & $11(19.3 \%)$ & $26(22.4 \%)$ & \\
\hline Histological grade & & & & 0.514 \\
\hline I-II & $118(68.2 \%)$ & $37(64.9 \%)$ & $81(69.8 \%)$ & \\
\hline III & $55(31.8 \%)$ & $20(35.1 \%)$ & $35(30.2 \%)$ & \\
\hline Surgery type & & & & 0.056 \\
\hline Mastectomy & $128(74.0 \%)$ & $37(64.9 \%)$ & $91(78.4 \%)$ & \\
\hline $\mathrm{BCS}$ & $45(26.0 \%)$ & $20(35.1 \%)$ & $25(21.6 \%)$ & \\
\hline Chemotherapy & & & & 0.097 \\
\hline Yes & $143(82.7 \%)$ & $51(89.5 \%)$ & $92(79.3 \%)$ & \\
\hline No & $30(17.3 \%)$ & $6(10.5 \%)$ & $24(20.7 \%)$ & \\
\hline Hormonal therapy & & & & 0.348 \\
\hline Yes & $137(79.2 \%)$ & $43(75.4 \%)$ & $94(81.0 \%)$ & \\
\hline No & $36(20.8 \%)$ & $14(24.6 \%)$ & $22(19.0 \%)$ & \\
\hline Radiotherapy & & & & 0.996 \\
\hline Yes & $82(47.4 \%)$ & $27(47.4 \%)$ & $55(47.4 \%)$ & \\
\hline No & $91(52.6 \%)$ & $30(52.6 \%)$ & $61(52.3 \%)$ & \\
\hline Target therapy & & & & 0.204 \\
\hline Yes & $25(14.5 \%)$ & $11(19.3 \%)$ & $14(12.1 \%)$ & \\
\hline No & $148(85.5 \%)$ & $46(80.7 \%)$ & $102(87.9 \%)$ & \\
\hline
\end{tabular}

BCS: breast conserving surgery; TNBC: triple-negative breast cancer. 
Table 3: ER, PR, and HER2 status or risk factors grouping with outcomes

\begin{tabular}{|c|c|c|c|c|c|c|c|}
\hline & \multirow[t]{2}{*}{ Total } & \multirow[t]{2}{*}{ Relapse } & \multirow[t]{2}{*}{ Died } & \multicolumn{2}{|c|}{ DFS } & \multicolumn{2}{|r|}{ OS } \\
\hline & & & & $P$ & HR(95\%CI) & $P$ & HR(95\%CI) \\
\hline $\begin{array}{l}\text { ER, PR, and } \\
\text { HER2 status }\end{array}$ & 122 & 43 & 29 & 0.002 & $1.779(1.232-3.701)$ & 0.002 & $2.356(1.825-4.857)$ \\
\hline $\begin{array}{l}\text { ER+, PR+, } \\
\text { HER2- }\end{array}$ & $59(48.4 \%)$ & $13(30.2 \%)$ & $6(20.7 \%)$ & & & & \\
\hline $\begin{array}{l}\text { ER+, PR+, } \\
\text { HER2+ }\end{array}$ & $24(19.7 \%)$ & $8(18.6 \%)$ & $7(24.1 \%)$ & & & & \\
\hline $\begin{array}{l}\mathrm{ER}+/ \mathrm{PR}+, \\
\text { HER2-/+ }\end{array}$ & $39(32.0 \%)$ & $22(51.2 \%)$ & $16(55.2 \%)$ & & & & \\
\hline $\begin{array}{l}\text { Risk factors } \\
\text { grouping }\end{array}$ & 173 & 59 & 42 & $<0.0013 .658$ & $(1.2570-6.239)$ & $<0.001$ & $3.852(2.084-5.962)$ \\
\hline 1 & $125(22.0 \%)$ & $27(45.8 \%)$ & $16(38.1 \%)$ & & & & \\
\hline 2 & $38(16.2 \%)$ & $22(37.3 \%)$ & $18(42.9 \%)$ & & & & \\
\hline 3 & $10(5.8 \%)$ & $10(16.9 \%)$ & $8(19.0 \%)$ & & & & \\
\hline
\end{tabular}

DFS: disease-free survival; OS: overall survival.

Table 4: Multivariate analysis of predictors with Cox model

\begin{tabular}{llll}
\hline Outcome & Variables & $\boldsymbol{P}$ & HR (95\%CI) \\
\hline DFS & Age (30-35) & 0.002 & $0.429(0.248-0.742)$ \\
& PR status (negative) & 0.042 & $1.757(1.019-3.021)$ \\
& Tumor grade (pT) & 0.012 & $1.843(1.612-3.669)$ \\
& Lymph nodes post-surgery (pN+) & $<0.001$ & $2.427(1.536-3.835)$ \\
ER, PR, and HER2 Status & 0.038 & $1.786(1.254-3.568)$ \\
OS & Risk factors grouping & 0.006 & $1.818(1.185-2.791)$ \\
& Age (30-35) & 0.002 & $0.354(0.185-0.678)$ \\
& PR status (negative) & 0.003 & $2.825(1.408-5.587)$ \\
& Lymph nodes post-surgery $(\mathrm{pN}+)$ & 0.002 & $2.493(1.410-4.409)$ \\
& Risk factors grouping & 0.010 & $1.953(1.171-3.259)$ \\
\hline
\end{tabular}

DFS: disease-free survival; OS: overall survival.

the risk of disease progression and death increasing by 1.818 and 1.953 times, respectively. This finding indicated that the risk grouping score may be helpful in selecting patient subgroups for further adjuvant treatment.

\section{MATERIALS AND METHODS}

\section{Patients and follow-up}

A total of 1796 patients with histologically confirmed breast cancer with surgical resection in West China Hospital of Sichuan University were confirmed from 2010 to 2012. Among these patients, a total of 173 patients younger than age 35 without distant metastasis at first diagnosis were included in this retrospective study. Patients were investigated and followed up every 3 months for 3 years, every 6 months for 5 years, and every 12 months in 6-10 years after operation. The examination of carcinoembryonic antigen (CEA) and carbohydrate antigen 153 (CA153), breast ultrasound, and mammography, liver ultrasound, chest X-ray, head CT scanning, and gynecological examination were included. This study was approved by the Research Ethics Committee of West China Hospital of Sichuan University.

\section{Pathology methods}

The expression of ER, PR, HER2, and Ki-67 were tested by immunohistochemical staining. The following primary antibodies were applied: monoclonal ER antibody (clone SP1; Ventana, Tucson, AZ, USA), monoclonal PR (clone 1E2; Ventana), Ki-67 (clone 309; Ventana), and HER2 (clone 4B5; Roche, Sandhofer, Mannheim, Germany). The cut-off value of positive ER/ PR was defined as $\geq 1 \%$ within immunoreactive tumor cell nuclei. The cut-off value of high Ki-67 was $\geq 14 \%$. The immunohistochemical staining for HER2 was scored as 0 , $1+, 2+$, or $3+; 3+$ was considered as HER 2 overexpression, 0 or $1+$ was defined as HER2 negative, and $2+$ was equivocal, fluorescence in situ hybridization (FISH) 
testing was needed for explicit HER2 gene amplification status. All staining specimens were independently viewed and scored by two pathologists in West China Hospital, Sichuan University.

\section{Molecular subtypes and treatment}

Molecular subtypes were classified as Luminal A $($ ER+ and/or PR+, HER2-, Ki-67 < 14), Luminal B (HER2-) (ER+ and/or PR+, HER2-, Ki-67 $\geq 14)$, luminal B (HER2+) (ER+ and/or PR+, HER2+, any Ki67), HER2-enriched (ER-, PR-, HER2+, any Ki-67) and triple-negative (ER-, PR-, HER2-, any Ki-67) breast cancer (TNBC) in accordance with the St Gallen expert consensus of 2013 [10]. Post-operative patients with hormone receptor positive and $\mathrm{Ki}-67<14 \%$ received adjuvant endocrine therapy for 5 years, while patients who were hormone receptor positive and Ki-67 $\geq 14 \%$ underwent chemotherapy and endocrine therapy. HER2+ patients received Trastuzumab and chemotherapy, while TNBC received chemotherapy. Patients who were positive for axillary lymph node post-surgery $(n \geq 3)$ or underwent breast conserving surgery received post-operative radiotherapy.

\section{Classification of hormone receptor and assessment of risk factor scores}

Breast cancer patients were treated according to ER and HER2 status in clinical setting. ER+ tumors typically respond to hormone therapy, whereas HER2+ tumors respond to anti-HER2 therapy. Given its nature as a highly heterogeneous disease with different histology, gene expression profiles, or mutation, hormone-positive breast cancer usually presents various clinical courses and responses to systemic treatment [3]. PR is another molecular marker that may be used in the clinic, as loss of $\mathrm{PR}$ in ER+ tumors is thought to be predictive for the lack of response to hormone therapy [11]. Besides, variability in Ki-67 scoring was observed in several of the world's most experienced laboratories and significant interobserver variability were detected due to limited analytical validity [12]. According to a previous study, we hereby classified hormone receptor-positive breast cancer as level 1 (ER+, $\mathrm{PR}+$, HER2-), level 2 (ER+, PR+, HER2 +$)$, or level 3 $(\mathrm{ER}+$ or $\mathrm{PR}+, \mathrm{HER} 2-/+)$ based on the ER, PR, and HER2 status and regardless of the expression of Ki-67 [3]. Patients with level 2 and level 3 tumors were considered to be at a more aggressive state and were treated with more chemotherapy than level 1 , whereas level 1 subgroup received more endocrine therapy than the other two levels.

A total of 173 patients were divided into three groups based on the scores of important risk factors including ER, PR, HER2, and Ki-67 status (ER-, PR-, and HER2+, and Ki-67+, one point each), tumor grade (grade 1 is considered as one point, and so on), and lymph nodes post-surgery ( 0 for no positive node, 1 for $1-3$ positive nodes, 2 for $4-9$ positive nodes, and 3 for $\geq 10$ positive nodes). Group 1 was scored $1-4$, group 2 was scored 5-6, and group 3 was scored 7-10, respectively.

\section{Statistical analysis}

DFS was defined as the time from diagnosis to the date of disease relapse, death, or last follow-up. OS was calculated from the time of diagnosis to death as a result of recurrence events or last follow-up, whichever occurred first. The follow-up deadline was March 2017. The relationship between the different age groups and the clinicopathological factors was analyzed by $\chi^{2}$ test. The end-points were estimated using the Kaplan-Meier method, and the differences between survival curves were tested using the log rank test. Univariate and multivariate analyses with the Cox proportional hazard regression model were performed to assess the influence of potential confounders on DFS and OS. The crude hazard ratios (HR) and corresponding 95\% confidence intervals (CIs) were reported. Statistical analyses were performed using the SPSS (version 20.0) software package (SPSS Inc., Chicago, IL, USA). $P<0.05$ was considered statistically significant.

\section{CONCLUSIONS}

In conclusion, this study shows that compared with their older counterparts, patients younger than 30 years at diagnosis with breast cancer constitute an independent risk factor for decreased DFS and OS among young patients ( $\leq 35$ years). Both PR status and lymph nodes involved post-surgery present clinical value as predictive factors of patient prognosis; moreover, we hope that the grading system based on hormone level and risk factor grouping may serve a useful index for evaluating the risk of breast cancer in young women to identify subgroups of patients with poor prognosis and offer therapeutic strategies for young breast cancer patients.

\section{ACKNOWLEDGMENTS}

We thank all practitioners who participated in the documentation of the data.

\section{CONFLICTS OF INTEREST}

The authors declare that there is no conflicts of interest.

\section{REFERENCES}

1. Wu J, Xue X, Zhang B, Cao H, Kong F, Jiang W, Li J, Sun D, Guo R. Enhanced antitumor activity and attenuated cardiotoxicity of Epirubicin combined with Paeonol against breast cancer. Tumour Biol. 2016; 37:12301-13. https://doi. org/10.1007/s13277-016-5088-9. 
2. Torre LA, Bray F, Siegel RL, Ferlay J, Lortet-Tieulent J, Jemal A. Global cancer statistics, 2012. CA Cancer J Clin. 2015; 65:87-108. https://doi.org/10.3322/caac.21262.

3. Zhao Y, Dong X, Li R, Song J, Zhang D. Correlation Between Clinical-Pathologic Factors and Long-Term FollowUp in Young Breast Cancer Patients. Transl Oncol. 2015; 8:265-72. https://doi.org/10.1016/j.tranon.2015.05.001.

4. Chen HL, Ding A, Wang FW. Prognostic effect analysis of molecular subtype on young breast cancer patients. Chin J Cancer Res. 2015; 27:428-36. https://doi.org/10.3978/j. issn.1000-9604.2015.08.02.

5. Azim HA, Partridge AH. Biology of breast cancer in young women. Breast Cancer Research. 2014; 16. https://doi.org/ Artn42710.1186/S13058-014-0427-5.

6. Lee HB, Han W. Unique features of young age breast cancer and its management. 2014; 17:301-7.

7. Anastasiadi Z, Lianos GD, Ignatiadou E, Harissis HV, Mitsis M. Breast cancer in young women: an overview. Updates Surg. 2017; 69:313-317. https://doi.org/10.1007/ s13304-017-0424-1.

8. Jia X, Liu G, Mo M, Cheng J, Shen Z, Shao Z. Reproductive factors and hormone receptor status among very young $(<35$ years) breast cancer patients. Oncotarget. 2015; 6:2457180. https://doi.org/10.18632/oncotarget.4698.

9. Anders CK, Fan C, Parker JS, Carey LA, Blackwell KL, Klauber-DeMore N, Perou CM. Breast carcinomas arising at a young age: unique biology or a surrogate for aggressive intrinsic subtypes? J Clin Oncol. 2011; 29:e18-20.

10. Goldhirsch A, Winer EP, Coates AS, Gelber RD, PiccartGebhart M, Thurlimann B, Senn HJ; Panel members. Personalizing the treatment of women with early breast cancer: highlights of the St Gallen International Expert Consensus on the Primary Therapy of Early Breast Cancer 2013. Ann Oncol. 2013; 24:2206-23. https://doi. org/10.1093/annonc/mdt303.

11. Creighton CJ. The molecular profile of luminal B breast cancer. Biologics. 2012; 6:289-97. https://doi.org/10.2147/ BTT.S29923.

12. Polley MY, Leung SC, McShane LM, Gao D, Hugh JC, Mastropasqua MG, Viale G, Zabaglo LA, PenaultLlorca F, Bartlett JM, Gown AM, Symmans WF, Piper T, et al. An international Ki-67 reproducibility study. J Natl Cancer Inst. 2013; 105:1897-906. https://doi. org/10.1093/jnci/djt306.

13. Gnerlich JL, Deshpande AD, Jeffe DB, Sweet A, White $\mathrm{N}$, Margenthaler JA. Elevated breast cancer mortality in women younger than age 40 years compared with older women is attributed to poorer survival in early-stage disease. J Am Coll Surg. 2009; 208:341-7. https://doi. org/10.1016/j.jamcollsurg.2008.12.001.

14. Maggard MA, O’Connell JB, Lane KE, Liu JH, Etzioni DA, Ko CY. Do young breast cancer patients have worse outcomes? Journal of Surgical Research. 2003; 113:109-13. https://doi.org/10.1016/s0022-4804(03)00179-3.
15. Anders CK, Hsu DS, Broadwater G, Acharya CR, Foekens JA, Zhang Y, Wang Y, Marcom PK, Marks JR, Febbo PG, Nevins JR, Potti A, Blackwell KL. Young age at diagnosis correlates with worse prognosis and defines a subset of breast cancers with shared patterns of gene expression. J Clin Oncol. 2008; 26:3324-30. https://doi.org/10.1200/ JCO.2007.14.2471.

16. Peng R, Wang S, Shi Y, Liu D, Teng X, Qin T, Zeng Y, Yuan Z. Patients 35 years old or younger with operable breast cancer are more at risk for relapse and survival: a retrospective matched case-control study. Breast. 2011; 20:568-73. https://doi.org/10.1016/j.breast.2011.07.012.

17. Ordu C, McGuire K, Alco G, Nur Pilanci K, Koksal UI, Elbüken F, Erdogan Z, Agacayak F, Ilgun S, Sarsenov D, Öztürk A, İğdem Ş, Okkan S, et al. The Prognostic Impact of Molecular Subtypes and Very Young Age on Breast Conserving Surgery in Early Stage Breast Cancer. Cureus. 2016; 8:e633. https://doi.org/10.7759/cureus.633.

18. Quan ML, Paszat LF, Fernandes KA, Sutradhar R, McCready DR, Rakovitch E, Warner E, Wright FC, Hodgson N, Brackstone M, Baxter NN. The effect of surgery type on survival and recurrence in very young women with breast cancer. J Surg Oncol. 2017; 115:122-30. https://doi.org/10.1002/jso.24489.

19. Voogd AC, Nielsen M, Peterse JL, Blichert-Toft M, Bartelink H, Overgaard M, van Tienhoven G, Andersen KW, Sylvester RJ, van Dongen JA. Differences in risk factors for local and distant recurrence after breast-conserving therapy or mastectomy for stage I and II breast cancer: pooled results of two large European randomized trials. J Clin Oncol. 2001; 19:1688-97. https://doi.org/10.1200/jco.2001.19.6.1688.

20. Vila J, Gandini S, Gentilini O. Overall survival according to type of surgery in young $(</=40$ years $)$ early breast cancer patients: A systematic meta-analysis comparing breast-conserving surgery versus mastectomy. Breast. 2015; 24:175-81. https://doi.org/10.1016/j.breast.2015.02.002.

21. Yuan XM, Wang N, Ouyang T, Yang L, Song MY, Lin BY, Xie YT, Li JF, Pan KF, You WC, Zhang L. Current status of diagnosis and treatment of primary breast cancer in beijing, 2008. Chin J Cancer Res. 2011; 23:38-42. https://doi. org/10.1007/s11670-011-0038-y.

22. Fan L, Strasser-Weippl K, Li JJ, St Louis J, Finkelstein DM, Yu KD, Chen WQ, Shao ZM, Goss PE. Breast cancer in China. Lancet Oncol. 2014; 15:e279-89. https://doi. org/10.1016/s1470-2045(13)70567-9.

23. Colleoni M, Rotmensz N, Robertson C, Orlando L, Viale G, Renne G, Luini A, Veronesi P, Intra M, Orecchia R, Catalano G, Galimberti V, Nole F, et al. Very young women $(<35$ years) with operable breast cancer: features of disease at presentation. Ann Oncol. 2002; 13:273-9.

24. Mohammed H, Russell IA, Stark R, Rueda OM, Hickey TE, Tarulli GA, Serandour AA, Birrell SN, Bruna A, Saadi A, Menon S, Hadfield J, Pugh M, et al. Progesterone receptor modulates ERalpha action in breast cancer. Nature. 2015; 523:313-7. https://doi.org/10.1038/nature14583. 
25. Bardou VJ, Arpino G, Elledge RM, Osborne CK, Clark GM. Progesterone receptor status significantly improves outcome prediction over estrogen receptor status alone for adjuvant endocrine therapy in two large breast cancer databases. J Clin Oncol. 2003; 21:1973-9. https://doi. org/10.1200/JCO.2003.09.099.

26. Perou CM, Sorlie T, Eisen MB, van de Rijn M, Jeffrey SS, Rees CA, Pollack JR, Ross DT, Johnsen H, Akslen LA, Fluge O, Pergamenschikov A, Williams C, et al. Molecular portraits of human breast tumours. Nature. 2000; 406:747-52. https://doi.org/10.1038/35021093.
27. Villarreal-Garza C, Mohar A, Bargallo-Rocha JE, LasaGonsebatt F, Reynoso-Noveron N, Matus-Santos J, Cabrera P, Arce-Salinas C, Lara-Medina F, Alvarado-Miranda A, Ramirez-Ugalde MT, Soto-Perez-de-Celis E. Molecular Subtypes and Prognosis in Young Mexican Women With Breast Cancer. Clin Breast Cancer. 2016; 17:e95-e102. https://doi.org/10.1016/j.clbc.2016.11.007.

28. Keegan TH, DeRouen MC, Press DJ, Kurian AW, Clarke CA. Occurrence of breast cancer subtypes in adolescent and young adult women. Breast Cancer Res. 2012; 14:R55. https://doi.org/10.1186/bcr3156. 